\title{
A Short Survey on Surfaces Endowed with a Canonical Principal Direction
}

\author{
Alev Kelleci, Mahmut Ergüt
}

Alev Kelleci: Firat University, Faculty of Science, Department of Mathematics, 23200 Elazı̆̆, Turkey., e-mail:alevkelleci@hotmail.com,

Mahmut Ergüt: Namı Kemal University, Faculty of Science and Letters, Department of Mathematics, 59030 Tekirda $\breve{g}$, Turkey, e-mail:mergut@nku.edu.tr

\begin{abstract}
In this paper, we would like to give a short survey of recent results on hypersurfaces with canonical principal direction relative to a fixed direction in a (semi-)Riemannian manifold. We also present some of our first results that we have recently obtained in this direction.
\end{abstract}

Keywords. Minkowski space · Lorentzian surfaces · canonical principal direction. Generalized constant ratio submanifolds.

MSC 2010 Classification. Primary: 53B25; Secondary:53B30 · 53C50.

\section{$1 \quad$ INTRODUCTION}

Let $\hat{M}$ be a (semi-)Riemannian manifold, $M$ a hypersurface of $\hat{M}$ and $X$ a vector field tangent to $\hat{M} . \quad M$ is said to have a canonical principal direction relative to $X$ if the tangential projection of $X$ to $M$ gives a principal direction. For example, a rotational hypersurface in Euclidean spaces has a canonical principal direction relative to a vector field parallel to its rotation axis, [12]. It turns out that when $\hat{M}$ is a product space $\tilde{M} \times \mathbb{R}$ or a semi-Euclidean space, some common interesting geometrical properties of hypersurfaces endowed with a canonical principal direction relative to $X$ occur if $X$ is chosen to be a fixed direction $k$ (See Theorem 3.6, Theorem 3.13, Theorem 3.15, Theorem 4.1 and Theorem 4.6).

Let $M^{n}(c), c= \pm 1$ denote the Riemannian space-form given by

$$
M^{n}(c)= \begin{cases}\mathbb{S}^{n} & \text { if } c=1, \\ \mathbb{H}^{n} & \text { if } c=-1 .\end{cases}
$$

We would like to note the following important property which relates constant angle surfaces to surfaces with a canonical principal direction. The projection $U$ of the unit vector field $T$ tangent to the second factor $\mathbb{R}$ to the tangential bundle of the surface is a principal direction for $M$ with the corresponding principal curvature equal to zero. Therefore, a constant angle surface in $M^{2}(c) \times \mathbb{R}$ is 
endowed with canonical principal direction relative to $T$. There are many classification results obtained so far, in different ambient spaces, [1, 3, 5, 6, 13, 15, 18].

A recent natural problem is that appears in the context of constant angle surfaces is to study those surfaces for which $\mathrm{U}$ remains a principal direction but the corresponding principal curvature is different from zero. This problem was studied in $\mathbb{S}^{2} \times \mathbb{R}[4]$ and $\mathbb{H}^{2} \times \mathbb{R}[7]$. Further, this problem has been recently studied in Euclidean spaces and semi-Euclidean spaces, (see in $[10,19,20]$ ) where $T$ is replaced by a constant direction $k$.

On the other hand, in $[8,9,11,23]$ authors study generalized constant ratio surfaces. A hypersurface $M$ in a semi-Euclidean space $\mathbb{E}_{t}^{n+1}$ is said to be a generalized constant ratio surfaces if the tangential component of its position vector is a principal direction of $M$. It is well-known that planes and complete hypersurfaces of $\mathbb{E}_{t}^{n+1}$ with constant sectional curvatures are trivial examples of generalized constant ratio surfaces.

This paper is organized as follows. In Sect. 2, we mention the notation that we use in this paper. In Sect. 3 and Sect.4, we present a short survey of recent results on surfaces endowed with a canonical principle curvatures. In Sect. 5, we show some of the results that we have recently obtained. In Sect. 6 we present classifications of generalized constant ratio hypersurfaces in Minkowski spaces.

\section{PRELIMINARIES}

In this section, we would like to give a brief summary of basic results on Lorentzian surfaces, (see for detail, [2, 21]).

Let $\mathbb{E}_{t}^{m}$ denote the semi-Euclidean $m$-space with the canonical semi-Euclidean metric tensor of index $t$ given by

$$
\tilde{g}=\sum_{i=1}^{m-t} d x_{i}^{2}-\sum_{j=m-t+1}^{m} d x_{j}^{2},
$$

where $x_{1}, x_{2}, \ldots, x_{m}$ are rectangular coordinates of the points of $\mathbb{E}_{t}^{m}$.

Let $\mathbb{S}_{t}^{n}\left(r^{2}\right)$ and $\mathbb{H}_{t-1}^{n}\left(-r^{2}\right)$ denote the de Sitter space-time and the hyperbolic space of dimension $n>2$ defined by

$$
\begin{aligned}
\mathbb{S}_{t}^{n}\left(1 / r^{2}\right) & =\left\{x \in \mathbb{E}_{t}^{n+1}:\langle x, x\rangle=r^{-2}\right\}, \\
\mathbb{H}_{t-1}^{n}\left(-1 / r^{2}\right) & =\left\{x \in \mathbb{E}_{t}^{n+1}:\langle x, x\rangle=-r^{-2}\right\} .
\end{aligned}
$$

For a short notation, we put $\mathbb{H}_{0}^{n}(-1)=\mathbb{H}^{n}$ and $\mathbb{S}_{0}^{n}(1)=\mathbb{S}^{n}$.

We would like to note that all further notations, basic definitions and basic facts that we will use in this paper are described in [8,23]. We also would like to refer to $[4,7,19,20]$ for detailed information of definition and geometrical interpretation of surfaces endowed with canonical principal direction. 

PRINCIPAL DIRECTION IN PRODUCT SPACES

In recent years, a lot of research has been done about $\tilde{M}^{2} \times \mathbb{R}$ by considering the unit vector field $T$ tangent to the second factor, parallel along $\tilde{M}^{2} \times \mathbb{R}$. A special case is when $\tilde{M}$ is a 2-dimensional Riemanmian space form, i.e., $\tilde{M}=$ $M^{2}(c), c= \pm 1$. A surface $M$ in $M^{2}(c) \times \mathbb{R}$ is said to be endowed with canonical principal direction (in short, CPD) if the projection of $T$, i.e. the canonical unit vector tangent to the $\mathbb{R}$-direction, onto the tangent space of $M$, is a principal direction. In this case, $T$ can be decomposed as

$$
T=\sin \theta U+\cos \theta N
$$

where $N$ is the unit normal vector field of surface $M$. Here, $S U=k_{1} U$ for a smooth function $k_{1}$ where $S$ is the shape operator of $M$ in $\mathbb{M}^{2} \times \mathbb{R}$, respectively. Note that we consider the case $\theta \notin\left\{0, \frac{\pi}{2}\right\}$ to eliminate trivial cases.

In this section, we would like to present a survey of classification results recently obtained. However, before we proceed, we would like to note that a further generalization of this notion is isometric immersions which belongs to the class $\mathcal{A}$. An isometric immersion $f: M \rightarrow \mathbb{S}^{n} \times \mathbb{R}$ is said to have this property if $U$ is an eigenvector of all shape operators of $f$, where $M$ is an $m$ dimensional submanifold of $\mathbb{S}^{n} \times \mathbb{R}$. This class was introduced in [22], where a complete description was given for hypersurfaces, and extended to submanifolds of $\mathbb{S}^{n} \times \mathbb{R}$ in [17].

\subsection{Surfaces in $\mathbb{S}^{2} \times \mathbb{R}$}

We may note that the study of CPD surfaces in $\mathbb{S}^{2} \times \mathbb{R}$ was investigated in [4]. The following results were obtained in that paper.

Let $M$ be a surface endowed with canonical principal direction in $\mathbb{S}^{2} \times \mathbb{R}$. By choosing an appropriate local coordinate system on $M$, one can see that the induced metric $g$ of $M$ becomes

$$
g=d x^{2}+\beta^{2}(x, y) d y^{2} .
$$

Moreover, the shape operator $S$ with respect to the basis $\left\{\frac{\partial}{\partial x}, \frac{\partial}{\partial y}\right\}$ is given by

$$
S=\left(\begin{array}{cc}
\theta_{x} & 0 \\
0 & \frac{\beta_{x} \tan \theta}{\beta}
\end{array}\right) .
$$

(See [4].)

Remark 3.1. An analogous result for CPD surfaces in $\mathbb{H}^{2} \times \mathbb{R}$ is obtained in [7].

First, we would like to give the following characterization for CPD surfaces in $\mathbb{S}^{2} \times \mathbb{R}$. 
Theorem 3.2. [5] Let $M$ be an immersed in $\mathbb{S}^{2} \times \mathbb{R}$ and $p$ a point of $M$ for which $\theta(p) \notin\{0, \pi / 2\}$. Then, $U$ is a principal direction if and only if $M$ considered as a surface in $\mathbb{E}^{4}$ is normally flat.

The following classification result is obtained in [4].

Proposition 3.3. [4] A surface $M$ immersed in $\mathbb{S}^{2} \times \mathbb{R}$ is a surface for which $U$ is a principal direction if and only if the immersion $F$ is in the neighborhood of a point $p$ where $\theta(p) \notin\left\{0, \frac{\pi}{2}\right\}$ given by

$$
\begin{aligned}
& F: M \quad \longrightarrow \mathbb{S}^{2} \times \mathbb{R} \\
& (x, y) \quad \longmapsto \quad\left(F_{1}(x, y), F_{2}(x, y), F_{3}(x, y), F_{4}(x)\right),
\end{aligned}
$$

where

$$
F_{j}(x, y)=\int_{y_{0}}^{y} \alpha_{j}(v) \sin (\psi(x)+\phi(v)) d v
$$

for $j=1,2,3, \psi^{\prime}(x)=\cos (\theta(x)), F_{4}^{\prime}(x)=\sin (\theta(x))$ and $\left(\alpha_{1}, \alpha_{2}, \alpha_{3}\right)$ is a curve in $\mathbb{S}^{2}$ and $F_{1}^{2}+F_{2}^{2}+F_{3}^{2}=1$. Moreover $\alpha_{1}, \alpha_{2}, \alpha_{3}, \psi$ and $\phi$ are functions on $M$ related by

$$
\begin{aligned}
\alpha_{j}^{\prime}(y)= & -\cos (\psi(x)+\phi(y)) \int_{y_{0}}^{y} \alpha_{j}(v) \cos (\psi(x)+\phi(v)) d v \\
& -\sin (\psi(x)+\phi(y)) \int_{y_{0}}^{y} \alpha_{j}(v) \sin (\psi(x)+\phi(v)) d v .
\end{aligned}
$$

A direct consequence of this proposition is

Corollary 3.4. [4] A surface $M$ immersed in $\mathbb{S}^{2} \times \mathbb{R}$ is a minimal surface with $U$ a principal direction if and only if the immersion $F$ is (up to isometries of $\mathbb{S}^{2} \times \mathbb{R}$ ) in the neighborhood of a point $p$ where $\theta \notin\left\{0, \frac{\pi}{2}\right\}$ given by

$$
\begin{aligned}
F: M \longrightarrow \mathbb{S}^{2} \times \mathbb{R}, \\
(x, y) \longmapsto\left(\frac{\sin x}{\sqrt{1+c^{2}}}, \frac{\sqrt{\cos ^{2} x+c^{2}} \cos y}{\sqrt{1+c^{2}}}, \frac{\sqrt{\cos ^{2} x+c^{2}} \sin y}{\sqrt{1+c^{2}}}, F_{4}(x)\right)
\end{aligned}
$$

with

$$
F_{4}(x)=\int_{0}^{x} \frac{c}{\sqrt{\cos ^{2}(u)+c^{2}}} d u .
$$

Corollary 3.5. [4] A surface $M$ immersed in $\mathbb{S}^{2} \times \mathbb{R}$ is a flat surface with $U$ a principal direction if and only if the immersion $F$ is (up to isometries of $\mathbb{S}^{2} \times \mathbb{R}$ ) in the neighborhood of a point $p$ where $\theta \notin\left\{0, \frac{\pi}{2}\right\}$ given by

$$
\begin{aligned}
& F: M \rightarrow \mathbb{S}^{2} \times \mathbb{R} \\
& (x, y) \longmapsto\left(\frac{\sqrt{1+d-x^{2}}}{\sqrt{1+d}}, \frac{x \cos y}{\sqrt{1+d}}, \frac{x \sin y}{\sqrt{1+d}}, F_{4}(x)\right)
\end{aligned}
$$

with

$$
F_{4}(x)=\int_{0}^{x} \frac{\sqrt{d-u^{2}}}{\sqrt{1+d-u^{2}}} d u
$$




\subsection{Surfaces in $\mathbb{H}^{2} \times \mathbb{R}$}

In [7], the authors studied CPD surfaces in $\mathbb{H}^{2} \times \mathbb{R}$.

Note that we have the following characterization.

Theorem 3.6. [7] Let $M$ be a surface isometrically immersed in $\mathbb{H}^{2} \times \mathbb{R}$ such that $\theta \notin 0$. $U$ is a principal direction if and only if $M$ is normally flat in $\mathbb{R}_{1}^{3} \times \mathbb{R}$.

Theorem 3.7. [7] If $F: M \rightarrow \mathbb{H}^{2} \times \mathbb{R}$ is an isometric immersion with $\theta \notin$ $\left\{0, \frac{\pi}{2}\right\}$, then $U$ is a principal direction if and only if $F$ is given by

$$
F(x, y)=\left(F_{1}(x, y), F_{2}(x, y), F_{3}(x, y), F_{4}(x)\right),
$$

with $F_{j}(x, y)=A_{j}(y) \sinh \phi(x)+B_{j}(y) \cosh \phi(x)$, for $j=1,2,3$ and $F_{4}(x)=$ $\int_{0}^{x} \sin (\theta(\tau) d \tau)$, where $\phi^{\prime}(x)=\cos (\theta)$. The six functions $A_{j}$ and $B_{j}$ are found in one of the following three cases.

- Case 1.

$$
\begin{aligned}
& A_{j}(y)=\int_{0}^{y} H_{j}(\tau) \cosh \psi(\tau) d \tau+c_{1 j}, \\
& B_{j}(y)=\int_{0}^{y} H_{j}(\tau) \sinh \psi(\tau) d \tau+c_{2 j}, \\
& H_{j}^{\prime}(y)=B_{j}(y) \sinh \psi(y)-A_{j}(y) \cosh \psi(y) ;
\end{aligned}
$$

- Case 2.

$$
\begin{aligned}
A_{j}(y) & =\int_{0}^{y} H_{j}(\tau) \sinh \psi(\tau) d \tau+c_{1 j}, \\
B_{j}(y) & =\int_{0}^{y} H_{j}(\tau) \cosh \psi(\tau) d \tau+c_{2 j}, \\
H_{j}^{\prime}(y) & =-A_{j}(y) \sinh \psi(y)+B_{j}(y) \cosh \psi(y) ;
\end{aligned}
$$

- Case 3.

$$
\begin{aligned}
& A_{j}(y)= \pm \int_{0}^{y} H_{j}(\tau) d \tau+c_{1 j}, \\
& B_{j}(y)=\int_{0}^{y} H_{j}(\tau) d \tau+c_{2 j}, \\
& H_{j}^{\prime}(y)=c_{2 j} \mp c_{1 j} ;
\end{aligned}
$$

where $H=\left(H_{1}, H_{2}, H_{3}\right)$ is a curve on the de Sitter space $\mathbb{S}_{1}^{2}, \psi$ is a smooth function on $M$ and $c_{1}=\left(c_{11}, c_{12}, c_{13}\right), c_{2}=\left(c_{21}, c_{22}, c_{23}\right)$ are constant vectors.

Remark 3.8. [7] In order to obtain a unified description, we note that in all cases $F$ is given by

$$
F(x, y)=\left(A(y) \sinh \phi(x)+B(y) \cosh \phi(x), \int_{0}^{x} \sin \theta(\tau) d \tau\right),
$$


where $A$ is a curve in $\mathbb{S}_{1}^{2}$ and $B$ is a curve in $\mathbb{H}^{2}$ orthogonal to $A$ such that the two speeds $A^{\prime}$ and $B^{\prime}$ are parallel. Denoting by $H$ the unit vector of their common direction, one has $H=A \otimes B$ and moreover

- $H$ is a space-like curve in the first case,

- $H$ is a time-like curve in the second case,

- $H$ is a light-like curve in the last case.

Theorem 3.9. [7] If $F: M \rightarrow \mathbb{H}^{2} \times \mathbb{R}$ is an isometric immersion with angle function $\theta \notin\left\{0, \frac{\pi}{2}\right\}$, then $U$ is a principal direction if and only if $F$ is locally given by

$$
F(x, y)=(A(y) \sinh \phi(x)+B(y) \cosh \phi(x), \chi(x)),
$$

where $A(y)$ is a curve in $\mathbb{S}_{1}^{2}$ and $B$ is a curve in $\mathbb{H}^{2}$, such that $\langle A, B\rangle=0, A^{\prime} \| B^{\prime}$ and where $(\phi(x), \chi(x))$ is a regular curve in $\mathbb{R}^{2}$. The angle function $\theta$ of $M$ depends only on $x$ and coincides with the angle function of the curve $(\phi, \chi)$. In particular, we can arc length reparametrize $(\phi, \chi)$; then $(x, y)$ are canonical cordinates and $\theta^{\prime}(x)=\kappa(x)$, the curvature of $(\phi, \chi)$.

Theorem 3.10. [7] Let $F: M \rightarrow \mathbb{H}^{2} \times \mathbb{R}$ is an isometric immersion with $\theta \notin\left\{0, \frac{\pi}{2}\right\}$. Then $M$ has $U$ as a principal direction if and only if $F$ is given by

$$
F(x, y)=\left(f(y) \cosh \phi(x)+N_{f}(y) \sinh \phi(x), \chi(x)\right),
$$

where $f(y)$ is a regular curve in $\mathbb{H}^{2}$ and $N_{f}(y)=\frac{f(y) \otimes f^{\prime}(y)}{\sqrt{\left\langle f^{\prime}(y), f^{\prime}(y)\right\rangle}}$ represents the normal of $f$. Moreover, $(\phi, \chi)$ is a regular curve in $\mathbb{R}^{2}$ and the angle function $\theta$ of this curve is the same as the angle function of the surface parametrized by $F$.

Consequently, authors obtained the following classification results by considering minimal and flat surfaces.

Corollary 3.11. [7] Let $M$ be a surface isometrically immersed in $\mathbb{H}^{2} \times \mathbb{R}$, with $\theta \notin\left\{0, \frac{\pi}{2}\right\}$. Then $M$ is minimal with $U$ a principal direction if and only if the immersion is, up to isometries of the ambient space, locally given by one of the next cases

- $F(x, y)=\left(\frac{b(x)}{\sqrt{1+c_{1}^{2}-c_{2}^{2}}}, \sinh y \frac{\sqrt{a^{2}(x)+1}}{\sqrt{1+c_{1}^{2}-c_{2}^{2}}}, \cosh y \frac{\sqrt{a^{2}(x)+1}}{\sqrt{1+c_{1}^{2}-c_{2}^{2}}}, \chi(x)\right)$,

- $F(x, y)=\left(\cos y \frac{\sqrt{a^{2}(x)+1}}{\sqrt{-1-c_{1}^{2}+c_{2}^{2}}}, \sin y \frac{\sqrt{a^{2}(x)+1}}{\sqrt{-1-c_{1}^{2}+c_{2}^{2}}}, \frac{b(x)}{\sqrt{-1-c_{1}^{2}+c_{2}^{2}}}, \chi(x)\right)$,

- $F(x, y)=\left(b(x) y, \frac{b(x)}{2}\left(1-y^{2}\right)-\frac{1}{2 b(x)}, \frac{b(x)}{2}\left(1+y^{2}\right)+\frac{1}{2 b(x)}, \chi(x)\right)$,

where $\chi(x)=\int_{0}^{x} \frac{1}{\sqrt{a^{2}(\tau)+1}} d \tau$, with $a(x)=c_{1} \cosh x+c_{2} \sinh x, b(x)=a^{\prime}(x)$ and $c_{1}, c_{2}$ are constants. 
Theorem 3.12. [7] Let $M$ be a surface in $\mathbb{H}^{2} \times \mathbb{R}$, with $\theta \notin\left\{0, \frac{\pi}{2}\right\}$. Then $M$ is flat with $U$ a principal direction if and only if the immersion $F$ is, up to isometries of the ambient space, given by

- $F(x, y)=\left(\frac{x}{\sqrt{1+c}} \cos y, \frac{x}{\sqrt{1+c}} \sin y, \frac{\sqrt{x^{2}+c+1}}{\sqrt{1+c}}, \chi(x)\right)$,

- $F(x, y)=\left(\frac{\sqrt{x^{2}+c+1}}{\sqrt{-1-c}}, \frac{x}{\sqrt{-1-c}} \sinh y, \frac{x}{\sqrt{-1-c}} \cosh y, \chi(x)\right)$,

- $F(x, y)=\left(x y, \frac{x}{2}\left(1-y^{2}\right)-\frac{1}{2 x}, \frac{x}{2}\left(1+y^{2}\right)+\frac{1}{2 x}, \chi(x)\right)$,

where $\chi(x)=\int_{0}^{x} \frac{\sqrt{\tau^{2}+c}}{\sqrt{\tau^{2}+c+1}} d \tau, c \in \mathbb{R}$.

\subsection{Surfaces in $M^{2}(c) \times \mathbb{R}_{1}$}

In [10], Fu and Nistor gave a partial classification of CPD surfaces by assuming that the fixed vector is time-like. In this case, the fixed vector is $k=(0,0,1)$ which is time-like.

Similar to previous case, let $U$ stand for the unit tangent vector on the direction of $k^{T}$.

Theorem 3.13. [10] Let $M$ be a space-like surface in Lorentzian product spaces $M^{2}(c) \times \mathbb{R}_{1}$. Then, $U$ is a principal direction if and only if $M$ is normally flat in $\mathbb{R}_{1}^{3}$ for $c=0, \mathbb{R}_{1}^{4}$ for $c=1, \mathbb{R}_{2}^{4}$ for $c=-1$.

Next, we would like to mention the following theorem obtained in [10] where authors assume $k=(0,0,1)$.

Theorem 3.14. [10, 20] Let $L: M \rightarrow M^{2}(c) \times \mathbb{R}_{1}$ be a space-like surface. Then, $U$ is a canonical principal direction for $M$ if and only if $M$ is parametrized as:

- If $c=1$, then $L: M \rightarrow \mathbb{S}^{2} \times \mathbb{R}_{1}$,

$$
L(x, y)=\left(\cos \phi(x) f(y)+\sin \phi(x) N_{f}(y), \chi(x)\right),
$$

where $f(y)$ is a regular curve on $\mathbb{S}^{2}$ and

$$
N_{f}(y)=\frac{f(y) \otimes f^{\prime}(y)}{\sqrt{\left\langle f^{\prime}(y), f^{\prime}(y)\right\rangle}}
$$

represents the normal of $f$.

- If $c=-1$, then $L: M \rightarrow \mathbb{H}^{2} \times \mathbb{R}_{1}$,

$$
L(x, y)=\left(\cosh \phi(x) f(y)+\sinh \phi(x) N_{f}(y), \chi(x)\right),
$$

where $f(y)$ is a regular curve in $\mathbb{S}^{2}$ and

$$
N_{f}(y)=\frac{f(y) \otimes f^{\prime}(y)}{\sqrt{\left\langle f^{\prime}(y), f^{\prime}(y)\right\rangle}}
$$

represents the normal of $f$. 
- If $c=0$, then we have $L: M \rightarrow \mathbb{R}_{1}^{3}$ is congruent to one of the following two surfaces.

1. $L(x, y)=(\cos y, \sin y, 0) \phi(x)-(0,0,1) \chi(x)+\gamma(v)$, where

$$
\gamma(v)=\left(\int_{0}^{y} \psi(\tau) \sin (\tau) d \tau,-\int_{0}^{y} \psi(\tau) \cos (\tau) d \tau, 0\right), \psi \in C^{\infty}(M) .
$$

2. $L(x, y)=\left(\cos y_{0}, \sin \left(y_{0}\right), 0\right) \phi(x)-(0,0,1) \chi(x)+\gamma_{0}(y)$, where

$$
\gamma_{0}(y)=\left(-\left(\sin y_{0}\right) y,\left(\cos y_{0}\right) y, 0\right)
$$

and $y_{0}$ is a real constant.

In all three cases $\phi(x)=\int_{x_{0}}^{x} \cosh \theta(\tau) d \tau$ and $\chi(x)=\int_{x_{0}}^{x} \sinh \theta(\tau) d \tau$.

Now, we give the following results obtained in [10] for Lorentzian surfaces with canonical principal direction. We note that they gave the partial classification of those surfaces in that paper.

Theorem 3.15. [10] Let $M$ be a Lorentzian surface in Lorentzian product spaces $M^{2}(c) \times \mathbb{R}_{1}$, and let $\theta$ be the hyperbolic angle function. Then, $U$ is a principal direction if and only if $M$ is normally flat in $\mathbb{R}_{1}^{3}$ for $c=0, \mathbb{R}_{1}^{4}$ for $c=1, \mathbb{R}_{2}^{4}$ for $c=-1$.

Theorem 3.16. [10, 7] Let $L: M \rightarrow M^{2}(c) \times \mathbb{R}_{1}$ be a Lorentzian surface and let $\theta \notin 0$ be the hyperbolic angle function. Then, $U$ is a canonical principal direction for $M$ if and only if $M$ is parametrized as:

- If $c=1$, then $L: M \rightarrow \mathbb{S}^{2} \times \mathbb{R}_{1}$ is

$$
L(x, y)=\left(\cos \chi(x) f(y)+\sin \chi(x) N_{f}(y), \phi(x)\right),
$$

where $f(y)$ is a regular curve on $\mathbb{S}^{2}$ and

$$
N_{f}(y)=\frac{f(y) \otimes f^{\prime}(y)}{\sqrt{\left\langle f^{\prime}(y), f^{\prime}(y)\right\rangle}}
$$

represents the normal of $f$.

- If $c=-1$, then $L: M \rightarrow \mathbb{H}^{2} \times \mathbb{R}_{1}$ is

$$
L(x, y)=\left(\cosh \chi(x) f(y)+\sinh \chi(x) N_{f}(y), \phi(x)\right),
$$

where $f(y)$ is a regular curve in $\mathbb{S}^{2}$ and

$$
N_{f}(y)=\frac{f(y) \otimes f^{\prime}(y)}{\sqrt{\left\langle f^{\prime}(y), f^{\prime}(y)\right\rangle}}
$$

represents the normal of $f$. 
- If $c=0$, then $L: M \rightarrow \mathbb{R}_{1}^{3}$

1. $L(x, y)=(\chi(x) \cos y, \chi(x) \sin y, \phi(x))+\gamma(y)$ where

$$
\gamma(y)=\left(-\int_{0}^{y} \psi(\tau) \sin (\tau) d \tau, \int_{0}^{y} \psi(\tau) \cos (\tau) d \tau, 0\right), \psi \in C^{\infty}(M) .
$$

2. $L(x, y)=\left(\chi(x) \cos y_{0}, \chi(x) \sin y_{0}, \phi(x)\right)+\gamma_{0} y$, where

$$
\gamma_{0}=\left(-\sin y_{0}, \cos y_{0}, 0\right)
$$

and $y_{0}$ is a real constant.

In all these cases $\phi(x)=\int_{x_{0}}^{x} \cosh \theta(\tau) d \tau$ and $\chi(x)=\int_{x_{0}}^{x} \sinh \theta(\tau) d \tau$.

We have the following corollaries of the previvous theorem.

Corollary 3.17. [10] The only flat Lorentz surfaces $M$ immersed in $\mathbb{E}_{1}^{3}$ endowed with a canonical principal direction are given by the cylindirical surfaces parametrized in the last case of Theorem 3.16.

Corollary 3.18. [10] The only minimal Lorentz surfaces $M$ immersed in $\mathbb{E}_{1}^{3}$ endowed with a canonical principal direction are given by the catenoids of the 3rd kind parametrized as:

$$
L(x, y)=\left(m \cos \frac{t}{m} \cos y, m \cos \frac{t}{m} \sin y, x\right), m \in \mathbb{R}\{0\} .
$$

\section{SURFACES ENDOWED WITH CANONICAL PRINCIPAL DIRECTION IN EUCLIDEAN AND SEMI-EUCLIDEAN SPACES}

A surface in a semi-Euclidean space $\mathbb{E}_{r}^{3}$ is said to be endowed with canonical principal direction (CPD) if there exists a fixed direction $k$ such that $S\left(k^{T}\right)=k_{1} k^{T}$, where $k^{T}$ denote the tangential component of $k$. In [19], Munteanu and Nistor studied surfaces with CPD in $\mathbb{E}^{3}$, while some classifications of such surfaces in the Minkowski space $\mathbb{E}_{1}^{3}$ is obtained in [20] for some cases.

\subsection{Surfaces in $\mathbb{E}^{3}$}

Let $M$ be a surface with CPD in $\mathbb{E}^{3}$. Note that by choosing an appropriate rotation in $\mathbb{E}^{3}$, we may assume $k=(0,0,1)$ and we denote $U=k^{T} /\left\|k^{T}\right\|$. We define $\theta$ by $k=\sin \theta U+\cos \theta N$. To eliminate trivial cases we consider a point $p \in M$ with $\theta(p) \notin\left\{0, \frac{\pi}{2}\right\}$.

Note that if $U$ is a principal direction, then we can choose a local coordinate system $(x, y)$ in a neighborhood of $p$ so that $\partial_{x}$ is in the direction of $U$ and the 
metric $g$ has the form $g=d x^{2}+\beta^{2}(x, y) d y^{2}$. Further, the shape operator $S$ is given by

$$
S=\left(\begin{array}{cc}
\theta_{x} & 0 \\
0 & \frac{\beta_{x} \tan \theta}{\beta}
\end{array}\right) .
$$

Moreover, $\theta$ and $\beta$ are related by $\frac{\beta_{x}}{\cos \theta}$ is independent of $x$ and $\theta_{y}=0,[19]$.

In [19] the following characterization theorem obtained.

Theorem 4.1. [19] Let $M$ be a surface in $\mathbb{E}^{3}$ and $\theta \notin 0$ be the angle function. Let $(x, y)$ be local coordinates on $M$ such that $\partial_{x}$ is the direction of $U$. Then, $U$ is a principal direction if and only if $\theta_{y}=0$.

Further, the classification of surfaces with $\mathrm{CPD}$ in $\mathbb{E}^{3}$ was given as following.

Theorem 4.2. [19] Let $M$ be a surface isometrically immersed in $\mathbb{E}^{3}$ and let $\theta \notin 0, \frac{\pi}{2}$ be as before. Then, $U$ is a canonical principal direction if and only if $M$ is given, up to isometries of $\mathbb{E}^{3}$, by one of the following cases:

- $r: M \rightarrow \mathbb{E}^{3}$,

$$
r(x, y)=\left(\phi(x)(\cos y, \sin y)+\gamma(y), \int_{0}^{x} \sin \theta(\tau) d \tau\right)
$$

with

$$
\gamma(y)=\left(-\int_{0}^{y} \psi(\tau) \sin (\tau) d \tau, \int_{0}^{y} \psi(\tau) \cos (\tau) d \tau\right),
$$

where $\psi$ is a smooth function on a certain interval $I$.

- $r: M \rightarrow \mathbb{E}^{3}, r(x, y)=\left(\phi(x) \cos \left(y_{0}\right), \phi(x) \sin \left(y_{0}\right), \int_{0}^{y} \sin \theta(\tau) d \tau+y\left(v_{0}\right)\right)$ with $v_{0}=\left(-\sin \left(y_{0}\right), \cos \left(y_{0}\right), 0\right), y_{0} \in \mathbb{R}$. Notice that these surfaces are cylinders. In both cases $\phi(x)$ denotes a primitive of $\cos \theta$.

Similar to Sect. 3, the classifications of minimal and flat surfaces follows from the previous theorem.

Corollary 4.3. [19] Let $M$ be a surface isometrically immersed in $\mathbb{E}^{3}$. Then $M$ is minimal surface with $U$ a principal direction if and only if the immersion is, up to isometries of the ambient space, given by

$$
r(x, y)=\left(\sqrt{x^{2}+c^{2}} \cos y, \sqrt{x^{2}+c^{2}} \sin y, c \log \left(x+\sqrt{x^{2}+c^{2}}\right)\right), c \in \mathbb{R} .
$$

Remark 4.4. [19] We notice that this surface can be obtained by rotating the catenary around the $z$-axis. Hence, the only minimal surface in Euclidean 3space with canonical principal direction is the catenoid.

Corollary 4.5. [19] Let $M$ be a surface isometrically immersed in $\mathbb{E}^{3}$ and let $\theta \notin 0, \frac{\pi}{2}$ be the angle function. Then $M$ is a flat surface with $U$ a principal direction if and only if the immersion is, up to isometries of the ambient space, given by

$$
r(x, y)=\left(\phi(x) \cos \left(y_{0}\right), \phi(x) \sin \left(y_{0}\right), \int_{0}^{x} \sin \theta(\tau) d \tau\right)+y v_{0}
$$

where $v_{0}=\left(-\sin y_{0}, \cos y_{0}, 0\right), y_{0} \in \mathbb{R}$, and $\phi(x)$ represents a primitive of $\cos \theta$. 


\subsection{Surfaces in $\mathbb{E}_{1}^{3}$}

On the other hand, some classification results for surfaces endowed with canonical principal direction in $\mathbb{E}_{1}^{3}$ were obtained in [20], where Nistor studied spacelike surfaces. In that paper, the author gave a classification of those surfaces by assuming that the fixed direction is time-like and the fixed vector $k$ is considered to be $k=(0,0,1)$.

Theorem 4.6. [20] Let $M$ be a space-like surface in $\mathbb{E}_{1}^{3}$ and $\theta \notin 0$ be the hyperbolic angle function. Let $(x, y)$ be local coordinates on $M$ such that $\partial_{x}$ is the direction of $U$. Then, $U$ is a principal direction if and only if $\theta_{y}=0$.

Theorem 4.7. [20] Let $M$ immersed in $\mathbb{E}_{1}^{3}$ be a space-like surface and $\theta \notin 0$ be the hyperbolic angle function. Then, $M$ has a principal direction if and only if $M$ is parametrized in the last case of Theorem 3.14.

Consequently, we mention following two theorems related with minimality and flatness.

Theorem 4.8. [20] The only maximal space-like surfaces in $\mathbb{E}_{1}^{3}$ with a canonical principal direction are catenoids of the 1st kind, parametrized in local coordinates $(x, y)$ as

$$
(x, y) \mapsto\left(\sqrt{x^{2}-c^{2}} \cos y, \sqrt{x^{2}-c^{2}} \sin y, c \ln \left(x+\sqrt{x^{2}-c^{2}}\right)\right), c \in \mathbb{R}\{0\} .
$$

Theorem 4.9. [20] The only flat space-like surfaces in $\mathbb{E}_{1}^{3}$ with a canonical principal direction are generalized cylinders, parametrized in local coordinates $(x, y)$ as

$$
(x, y) \mapsto \sigma(x)+v_{0} y,
$$

where $\sigma(x)=\left(\cos y_{0} \int_{0}^{x} \cosh \theta(\tau) d \tau, \sin y_{0} \int_{0}^{x} \cosh \theta(\tau) d \tau,-\int_{0}^{x} \sinh \theta(\tau) d \tau\right), v_{0}=$ $\left(-\sin y_{0}, \cos y_{0}, 0\right), y_{0} \in \mathbb{R}$, and $\theta \notin 0$ denotes the hyperbolic angle function.

Remark 4.10. [20] The flat space-like surfaces endowed with a canonical principal direction classified in Theorem 4.9 are given by the generalized cylinders from the last case of Theorem 3.14. More precisely, these surfaces are cylinders over space-like curves with space-like rulings orthogonal to $k=(0,0,1)$.

$$
5 \text { NEW EXAMPLES OF SURFACES IN } \mathbb{E}_{1}^{3}
$$

In this section we would like to present some new examples of Lorentzian surface endowed with CPD in the Minkowski 3-space. Before we proceed, we would like to note that if $M$ is space-like, then its shape operator $S$ is diagonalizable, i.e., there exists a local orthonormal frame field $\left\{e_{1}, e_{2} ; N\right\}$ such that $S e_{i}=$ $k_{i} e_{i}, \quad i=1,2, \ldots, n$. In this case, the vector field $e_{i}$ and smooth function $k_{i}$ are called a principal direction and a principal curvature of $M$.

On the other hand, if $M$ is Lorentzian, then its shape operator can be nondiagonalizable. In this case, if all of the eigenvalues of $S$ are real at any point 
of $M$, then the matrix representation of $S$ with respect to a suitable pseudoorthonormal frame field $\left\{f_{1}, f_{2} ; N\right\}$ such that

$$
\left\langle f_{i}, f_{j}\right\rangle=\delta_{i j}-1, \quad i, j=1,2
$$

of the tangent bundle of $M$, the shape operator of a Lorentzian surface in $\mathbb{E}_{1}^{3}$ can be assumed to be one of canonical forms given by

$$
\begin{gathered}
\text { Case 1. } S=\operatorname{diag}\left(k_{1}, k_{2}\right), \quad \text { Case } 2 . \quad S=\left(\begin{array}{cc}
k_{1} & \mu \\
0 & k_{1}
\end{array}\right) \\
\text { Case 3. } S=\left(\begin{array}{cc}
k_{1} & \mu \\
-\mu & k_{1}
\end{array}\right)
\end{gathered}
$$

for some smooth functions $k_{1}, k_{2}$ and a non-vanishing function $\mu$, where the frame field is chosen to be orthonormal in Case 1 and Case 3 and pseudoorthonormal in Case 2 (See for example [16]). We note that if the shape operator of $M$ is as given in Case 3 of (5.1), then $S$ has no eigenvalue. So, we will consider surfaces whose shape operator is as given in Case 1 or Case 2 of (5.1).

A null curve $\beta(s)$ in $\mathbb{E}_{1}^{3}$ is said to have a Cartan frame if there exists vector fields $\{A, B, C\}$ on $\beta$ such that $\langle A, A\rangle=\langle B, B\rangle=0,\langle A, B\rangle=-1,\langle A, C\rangle=$ $\langle B, C\rangle=0$ and $\langle C, C\rangle=1$ with $\beta^{\prime}=A, A^{\prime}=k_{1}(s) C$ and $B^{\prime}=k_{2} C$ for a constant $k_{2}$ and a smooth function $k_{1}$ which is vanishing only on a subset $\mathcal{U}$ with $\operatorname{int} U=\emptyset$. Then, the surface $M$ given by

$$
f(s, t)=\beta(s)+t B(s)
$$

is said to be a $B$-scroll. Note that in [16], M. Magid have proved that a surface in $\mathbb{E}_{1}^{3}$ with non-diagonalizable shape operator is isoparametric if and only if it is a $B$-scroll.

Example 5.1. [14] Consider the $B$-scroll given by

$$
c(\hat{s}, t)=\left(\frac{\hat{s}^{2}}{2}+t, \frac{(2 \hat{s}-1)^{3 / 2}}{3}, \frac{\hat{s}^{2}}{2}-\hat{s}+t\right) .
$$

It turns out that the shape operator of this surface with respect to the pseudoorthonormal frame field $\left\{\partial_{t}, \partial_{s}\right\}$ is

$$
S=\left(\begin{array}{cc}
0 & \mu \\
0 & 0
\end{array}\right)
$$

Moreover, it is a surface endowed with a canonical principal direction relative to $k=(1,0,0)$.

Further, we have recently obtained the following result.

Proposition 5.2. A flat minimal surfaces in $\mathbb{E}_{1}^{3}$ endowed with a canonical principal direction relative to a fixed direction is either an open part of a plane or congruent to the surface given in (5.3). 
Remark 5.3. By considering the above example, the problem of considering surfaces with shape operator given in Case 2 of (5.1) in terms of having canonical principal direction arises. Authors would like to announce that they have recently completed the classification of such surfaces with a canonical principal direction relative to a fixed direction in $\mathbb{E}_{1}^{3}$.

Example 5.4. [14] Consider the rotational surface with a light-like rotational axis in $\mathbb{E}_{1}^{3}$ given by

$$
x(s, t)=\left(\frac{1}{2} s t^{2}+s+\phi(s), s t, \frac{1}{2} s t^{2}+\phi(s)\right)
$$

for a smooth function $\phi$. It is well-kown that the principal directions of $M$ are

$$
e_{1}=\frac{1}{\sqrt{\varepsilon_{1}\left(-2 \phi^{\prime}-1\right)}} \partial_{s}, \quad e_{2}=\frac{1}{s} \partial_{t} .
$$

Further, we have

$$
(1,0,1)=\psi\left(e_{1}-N\right)
$$

for a smooth function $\psi$. Hence, the surface given by (5.4) is endowed with a canonical principal direction relative to $k=(1,0,1)$.

Remark 5.5. A direct computation yields that the surface given by (5.4) is minimal if and only if

$$
\frac{\phi^{\prime \prime}}{\left(2 \phi^{\prime}+1\right)}=\frac{1}{s}
$$

On the other hand, the surface given by (5.4) is flat if and only if $\phi$ is linear.

Remark 5.6. Authors also would like to announce that they have recently completed the classification of surfaces with a canonical principal direction relative to a fixed light-like direction in $\mathbb{E}_{1}^{3}$. IN $\mathbb{E}_{1}^{3}$

Generalized constant ratio surfaces in Euclidean spaces are firstly investigated in $[9,23]$. By definition, let $M$ be a surface in the ambient space, $x$ its position vector and $\theta$ denote the angle function between $x$ and the unit normal vector field $N$ of $M$. If the tangential part of $x$ is one of its principal directions, then $M$ is said to be a generalized constant ratio (in short, GCR surfaces). Note that, we would like to remember two following definition. The time-like cone of $\mathbb{E}_{1}^{3}$ is defined as the set of all time-like vectors of $\mathbb{E}_{1}^{3}$, that is,

$$
T=\left\{x \in \mathbb{E}_{1}^{3}:\langle x, x\rangle<0\right\} .
$$

The space-like cone of $\mathbb{E}_{1}^{3}$ is defined as the set of all space-like vectors of $\mathbb{E}_{1}^{3}$, that is,

$$
S=\left\{x \in \mathbb{E}_{1}^{3}:\langle x, x\rangle>0\right\} .
$$


In this section, we just would like to present classification of GCR surfaces in Minkowski 3-space obtained in [9, 8, 24]. Note that in [8], authors studied this surface independently from the paper at [24]. Fistly, we would like to give results for this surface obtained in [9].

\subsection{Lorentzian surfaces in $\mathbb{E}_{1}^{3}$}

Most recently, Lorentzian GCR surfaces in the 3-dimensional Minkowski space investigated by Fu and Yang in [11].

Theorem 6.1. [11] Let $x: M \rightarrow \mathbb{E}_{1}^{3}$ be a surface immersed in the 3-dimensional Minkowski space $\mathbb{E}_{1}^{3}$. If the immersion $x$ lies in the space-like cone, then $M$ is a $G C R$ surface if and only if the immersion $x(M)$ is given by one of the following eight statements holds:

- $x(s, t)=s(\cos u(s), \sin u(s) \cosh t, \sin u(s) \sinh t)$, where $u(s)=\int \frac{\cot \theta(s)}{s} d s ;$

- $x(s, t)=s(\sin u(s), \cos u(s) \cosh t, \cos u(s) \sinh t)$, where $u(s)=\int \frac{\cot \theta(s)}{s} d s ;$

- $x(s, t)=s\left(\cos u(s) f(t)+\sin u(s) f(t) \times f^{\prime}(t)\right)$, where $f$ is a time-like unit speed curve on $\mathbb{S}_{1}^{2}$ satisfying $\left(f, f^{\prime}, f^{\prime \prime}\right) \notin 0, u(s)=\int \frac{\cot \theta(s)}{s} d s ;$

- $x(s, t)=\frac{s}{2}\left(-e^{-u(s)}+e^{u}(s)\left(t^{2}-1\right), 2 e^{u}(s) t,-e^{-u(s)}+e^{u(s)}\left(t^{2}+1\right)\right)$, where $u(s)=\int \frac{\operatorname{coth} \theta(s)}{s} d s ;$

- $x(s, t)=\frac{s}{2}\left(-e^{u(s)}+e^{-u(s)}\left(t^{2}-1\right), 2 e^{-u(s)} t,-e^{u(s)}+e^{-u(s)}\left(t^{2}+1\right)\right)$, where $u(s)=\int \frac{\operatorname{coth} \theta(s)}{s} d s$;

- $x(s, t)=s(\cosh u(s) \cos t, \sinh u(s) \sin t, \sinh u(s))$, where

$$
u(s)=\int \frac{\operatorname{coth} \theta(s)}{s} d s
$$

- $x(s, t)=s(\cosh u(s), \sinh u(s) \sinh t, \sinh u(s) \cosh t)$, where

$$
u(s)=\int \frac{\operatorname{coth} \theta(s)}{s} d s
$$

- $x(s, t)=s\left(\cosh u(s) f(t)+\sinh u(s) f(t) \times f^{\prime}(t)\right)$, where $f$ is a time-like unit speed curve on $\mathbb{S}_{1}^{2}$ satisfying $\left(f, f^{\prime}, f^{\prime \prime}\right) \notin 0, u(s)=\int \frac{\operatorname{coth} \theta(s)}{s} d s$.

Further, if $x$ lies in the time-like cone, the following classification theorem was obtained.

Theorem 6.2. [11] Let $x: M \rightarrow \mathbb{E}_{1}^{3}$ be a surface immersed in the 3-dimensional Minkowski space. If the immersion $x$ lies in the timelike cone, then $M$ is a GCR surface if and only if the immersion $x(M)$ is given by one of the following five statements holds: 
- $x(s, t)=\frac{s}{2}\left(e^{-u(s)}+e^{u(s)}\left(t^{2}-1\right), 2 e^{u(s)} t, e^{-u(s)}+e^{u(s)}\left(t^{2}+1\right)\right)$, where

$$
u(s)=\int \frac{\tanh \theta(s)}{s} d s ;
$$

- $x(s, t)=\frac{s}{2}\left(e^{u(s)}+e^{-u(s)}\left(t^{2}-1\right), 2 e^{-u(s)} t, e^{u(s)}+e^{-u(s)}\left(t^{2}+1\right)\right)$, where $u(s)=\int \frac{\tanh \theta(s)}{s} d s$;

- $x(s, t)=s(\sinh u(s), \cosh u(s) \sinh t, \cosh u(s) \cosh t)$, where

$$
u(s)=\int \frac{\tanh \theta(s)}{s} d s ;
$$

- $x(s, t)=s(\sinh u(s) \sin t, \sinh u(s) \cos t, \cosh u(s))$, where

$$
u(s)=\int \frac{\tanh \theta(s)}{s} d s ;
$$

- $x(s, t)=s\left(\cosh u(s) f(t)+\sinh u(s) f(t) \times f^{\prime}(t)\right)$, where $f$ is a unit speed curve on $\mathbb{H}^{2}$ satisfying $\left\langle f^{\prime \prime}, f^{\prime \prime}\right\rangle \notin-\left\langle f, f^{\prime \prime}\right\rangle^{2}, u(s)=\int \frac{\tanh \theta(s)}{s} d s$.

We would like to also note the following consequences of the previous theorems.

Corollary 6.3. [11] A flat Lorentz $G C R$ surface in $\mathbb{E}_{1}^{3}$ is an open part of a plane or of a cylinder.

Corollary 6.4. [11] A Lorentzian GCR surface in $\mathbb{E}_{1}^{3}$ with constant mean curvature is a surface of revolution.

\subsection{Space-like GCR Surfaces in Minkowski 3-Space}

In [8] and [24], the authors independently studied the space-like GCR surface in Minkowski spaces. After, they independently obtained the complete classification of GCR surfaces in the Minkowski 3-space. All the following results obtained for space-like GCR surfaces in Minkowski spaces were given in [8, 24].

Theorem 6.5. [8] Let $M$ be a non-degenerated hypersurface in $\mathbb{E}_{1}^{n+1}$ with position vector $x$. If $M$ is GCR, then the tangential part of $x$ is either space-like or time-like.

Proposition 6.6. [8] Let $M$ be an oriented hypersurface in the Minkowski space $\mathbb{E}_{1}^{n+1}$ and $x$ its position vector. Consider a unit tangent vector field $e_{1}$ in the direction of $x^{T}$. Then, $M$ is a GCR hypersurface if and only if a curve $\alpha$ is a geodesic of $M$ whenever it is an integral curve of $e_{1}$.

Proposition 6.7. [8, 24] Let $M$ be a space-like hypersurface in the Minkowski space $\mathbb{E}_{1}^{n+1}$ and $x: M \rightarrow \mathbb{E}_{1}^{n+1}$ the position vector with the tangential component $x^{T}$. Then $M$ is GCR hypersurface if and only if $Y(\theta)=0$, whenever $\left\langle Y, x^{T}\right\rangle=0$, where $\theta$ is the angle function. 
First we assume that the surface is contained in the time-like cone.

Theorem 6.8. [8, 24] Let $x: M \rightarrow \mathbb{E}_{1}^{3}$ be a space-like surface immersed in the 3-dimensional Minkowski space. Also, assume that $M$ is lying in the time-like cone of $\mathbb{E}_{1}^{3}$. Then, $M$ is GCR if and only if it can be parametrized by

$$
x(s, t)=s\left(\cosh u(s) \varphi(t)+\sinh u(s) \varphi(t) \wedge \varphi^{\prime}(t)\right),
$$

where $\varphi=\varphi(t)$ is an arc-length parametrized curve lying on $\mathbb{H}^{2}(-1)$ and $u=$ $u(s)$ is a smooth function. In this case, $x$ can be decomposed as

$$
x=-s\left(\sinh \theta e_{1}+\cosh \theta N\right)
$$

for the function $\theta$ given by

$$
\operatorname{coth} \theta=s u^{\prime}
$$

Now, we will give the classification of space-like GCR surfaces in case the image of the immersion $x$ lies in the space-like cone.

Theorem 6.9. [8, 24] Let $x: M \rightarrow \mathbb{E}_{1}^{3}$ be a space-like surface immersed in the 3-dimensional Minkowski space. Also, assume that $M$ is lying in the space-like cone of $\mathbb{E}_{1}^{3}$. Then, $M$ is GCR if and only if it can be parametrized by

$$
x(s, t)=s\left(\cosh u(s) \varphi(t)+\sinh u(s) \varphi(t) \wedge \varphi^{\prime}(t)\right)
$$

where $\varphi=\varphi(t)$ is an arclength parametrized curve lying on $\mathbb{S}_{1}^{2}(1)$ and $u=u(s)$ is a smooth function. In this case, $x$ can be decomposed as

$$
x=s\left(\cosh \theta e_{1}+\sinh \theta N\right)
$$

for the function $\theta$ given by

$$
\tanh \theta=s u^{\prime} .
$$

As a direct corollary of the previous theorems, we have

Corollary 6.10. [8] A space-like rotational surface given by

$$
x(s, t)=(s \cosh u \cosh t, s \cosh u \sinh t, s \sinh u)
$$

or

$$
x(s, t)=(s \cosh u \sinh t, s \cosh u \cosh t, s \sinh u)
$$

is a GCR surface, where $u=u(s)$ is a non-vanishing smooth function.

Theorem 6.11. [8, 24] The flat space-like GCR surfaces immersed in $\mathbb{E}_{1}^{3}$ are an open parts of a plane or of a cylinder.

Proposition 6.12. [24] The space-like GCR surfaces with constant mean curvature immersed in $\mathbb{E}_{1}^{3}$ are surfaces of revolution. 


\section{ACKNOWLEDGMENTS}

This paper is a part of $\mathrm{PhD}$ thesis of the first author who is supported by The Scientific and Technological Research Council of Turkey (TUBITAK) as a $\mathrm{PhD}$ scholar. The authors would like to thank the referees for their valuable comments which helped to improve the manuscript.

\section{REFERENCES}

[1] Cermelli, P., Di Scala, A. J., Constant-angle surfaces in liquid crystals, Philosophical Magazine 87 (2007), 1871-1888.

[2] Chen, B.-Y., Pseudo-Riemannian Geometry, $\delta$-Invariants and Applications, World Scientific, Hackensack, NJ, 2011.

[3] Di Scala, A. J., Ruiz-Hernandez, G., Helix submanifolds of euclidean spaces, Monatsh Math. 157 (2009), 205-215 .

[4] Dillen, F., Fastenakels, J., Van der Veken, J., Surfaces in $\mathbb{S}^{2} \times \mathbb{R}$ with a canonical principal direction, Ann. Global Anal. Geom. 35 (2009), 381-396.

[5] Dillen, F., Fastenakels, J., Van der Veken, J., Vrancken, L., Constant angle surfaces in $\mathbb{S}^{2} \times \mathbb{R}$, Monaths Math. 152 (2007), 89-96.

[6] Dillen, F., Munteanu, M. I., Constant angle surfaces in $\mathbb{H}^{2} \times \mathbb{R}$, Bull. Braz. Math. Soc. New Series 40 (2009), 85-97.

[7] Dillen, F., Munteanu, M. I., Nistor, A. I., Canonical coordinates and principal directions for surfaces in $\mathbb{H}^{2} \times \mathbb{R}$ Taiwanese J. Math., 15 (2011), 2265-2289.

[8] Ergut, M., Kelleci, A., Turgay, N. C., On space-like generalized constant ratio hypersufaces in Minkowski spaces, see arXiv:1603.08415v1.

[9] Fu, Y., Munteanu, M. I., Generalized constant ratio surfaces in $\mathbb{E}^{3}$, Bull. Braz. Math. Soc. New Series 45 (2014), 73-90.

[10] Fu, Y., Nistor, A. I., Constant Angle Property and Canonical Principal Directions for Surfaces in $\mathbb{M}^{2}(c) \times \mathbb{R}_{1}$, Mediterr. J. Math. 10 (2013), 10351049.

[11] Fu, Y., Yang, D. On Lorentz GCR surfaces in Minkowski 3-space, Bull. Korean Math. Soc. 53 (2016), 227-245.

[12] Garnica, E., Palmas, O., Hernandez, G. R., Hypersurfaces with a canonical principal direction, Differential Geom. Appl 30 (2012), 382-391.

[13] Guler, F., Saffak, G., Kasap, E., Timelike Constant Angle Surfaces in Minkowski Space $\mathbb{R}_{1}^{3}$, Int. J. Contemp. Math. Sciences 6 (2011), 2189-2200. 
[14] Kim, Y. H., Turgay, N. C., On the Gauss map of surfaces in $\mathbb{E}_{1}^{3}$ concerning Cheng-Yau operator, J Korean Math Soc. 54 (2017), 381-397.

[15] Lopez, R., Munteanu, M. I., Constant angle surfaces in Minkowski space, Bull. Belg. Math. Soc. Simon Stevin 18 (2011), 271-286.

[16] Magid, M. A., Lorentzian isoparametric hypersurfaces, Pacific J. Math. 118 (1985), 165-197.

[17] Mendonça, B., Tojeiro, R., Umbilical submanifolds of $\mathbb{S}^{n} \times \mathbb{R}$, Canad. J. Math. 66 (2014), 400-428.

[18] Munteanu, M. I., Nistor, A. I., A new approach on Constant Angle Surfaces in $\mathbb{E}^{3}$, Turk J. Math 33 (2009), 169-178.

[19] Munteanu, M. I., Nistor, A. I., Complete classification of surfaces with a canonical principal direction in the Euclidean space $\mathbb{E}^{3}$, Cent. Eur. J. Math. 9 (2011), 378-389.

[20] Nistor, A. I., A note on spacelike surfaces in Minkowski 3-space Filomat 27 (2013), 843-849.

[21] O'Neill, B., Semi-Riemannian Geometry with Applications to Relativity. World Scientific, New York, 1983.

[22] Tojeiro, R., On a class of hypersurfaces in $\mathbb{S}^{n} \times \mathbb{R}$ and $\mathbb{H}^{n} \times \mathbb{R}$, Bull. Braz. Math. Soc. New Series 41 (2010), 199-209.

[23] Turgay, N. C., Generalized constant ratio hypersurfaces in Euclidean spaces (submitted) see arXiv:1504.07757.

[24] Yang, D., Fu, Y., Li, L., Geometry of space-like generalized constant ratio surfaces in Minkowski 3-space, Front. Math. China 12 (2017), 459-480. 